\title{
Experimental investigation of multibunch, multipass beam breakup in the Jefferson Laboratory Free Electron Laser Upgrade Driver
}

\author{
David R. Douglas, Kevin C. Jordan, Lia Merminga, Eduard G. Pozdeyev, Christopher D. Tennant,* and Haipeng Wang \\ Thomas Jefferson National Accelerator Facility, Newport News, Virginia 23606, USA
}

Todd I. Smith

W.W. Hansen Experimental Physics Laboratory, Stanford, California 94305, USA

Stefan Simrock

Deutsches Elektronen-Synchrotron, Notkestrasse 85, 22603 Hamburg, Germany

Ivan V. Bazarov and Georg H. Hoffstaetter

Laboratory for Elementary Particle Physics, Cornell University, Ithaca, New York 14853, USA

(Received 21 March 2006; published 27 June 2006)

\begin{abstract}
In recirculating accelerators, and, in particular, energy-recovery linacs, the maximum current can be limited by multipass, multibunch beam breakup (BBU), which occurs when the electron beam interacts with the higher-order modes (HOMs) of an accelerating cavity on the accelerating pass and again on the energy recovering pass. This effect is of particular concern in the design of modern high average current energy-recovery accelerators utilizing superconducting rf technology. Experimental characterization and observations of the instability at the Jefferson Laboratory $10 \mathrm{~kW}$ free electron laser (FEL) are presented. Measurements of the threshold current for the instability are made under a variety of beam conditions and compared to the predictions of several BBU simulation codes. This represents the first time in which the codes have been experimentally benchmarked. With BBU posing a threat to high current beam operation in the FEL driver, several suppression schemes were developed. These include direct damping of the dangerous HOM using cavity feedback and modifying the electron beam optics so as to reduce the coupling between the beam and mode. Both methods were shown to increase the threshold current for stability. Beam optical suppression techniques, in particular, have proved to be so effective that they are routinely used in the normal operations of the FEL Upgrade Driver.
\end{abstract}

DOI: 10.1103/PhysRevSTAB.9.064403

PACS numbers: 29.27.Bd

\section{INTRODUCTION}

Energy recovering linacs (ERLs) that utilize superconducting radio-frequency (SRF) technology offer an attractive alternative as drivers for, among other things, synchrotron light sources and free electron lasers (FEL), as they combine the desirable characteristics of both storage rings (high efficiency) and linear accelerators (superior beam quality) [1]. With the demand for high average current ERLs, one of the primary challenges is to provide adequate damping of the higher-order modes (HOMs). If not sufficiently damped, dipole HOMs can drive the multipass beam breakup (BBU) instability. This effect is of particular concern in the design of modern high average current energy-recovery accelerators utilizing SRF technology due to the relatively high quality factors of HOMs. Because BBU imposes a real threat to the operation of high current ERLs, a better understanding of the instability and development of suppression techniques are vitally important.

\footnotetext{
*Corresponding author. Electronic address: tennant@jlab.org
}

Beam breakup has already been observed at Jefferson Laboratory's FEL Upgrade Driver and preliminary measurements to characterize the instability were reported in a previous work [2]. The Driver is an energy-recovery based linear accelerator used to condition an electron beam for high-power lasing. Electrons are injected at $7 \mathrm{MeV}$ and are accelerated to $88 \mathrm{MeV}$ through a linac consisting of three cryomodules (each containing 8 superconducting niobium cavities). The beam is transported to a wiggler where up to $10 \mathrm{~kW}$ of laser power is generated [3]. The spent electron beam is recirculated and decelerated through the linac on the second pass. Upon exiting the linac, the $7 \mathrm{MeV}$ energy recovered beam is extracted to a dump.

The middle cryomodule in the linac incorporates a new 7-cell cavity design with the capability of achieving higher accelerating gradients, while the two adjacent cryomodules utilize standard continuous electron beam accelerator facility (CEBAF) 5-cell cavities. From rf measurements and subsequent simulations, it was determined that the new 7-cell cavity cryomodule (henceforth referred to as zone 3 ) contained dangerous HOMs that could facilitate BBU at currents below the nominal operating current. This was confirmed with observations of BBU in 2004 consistent with the predictions of simulations [2]. 
The mechanism for BBU begins when a beam bunch passes through a cavity off-axis, thereby exciting dipole higher-order modes. The magnetic field of an excited mode acts to deflect following bunches traveling through the cavity. Depending on the details of the machine optics, after recirculation the deflection produced by the mode translates into a transverse displacement at the cavity. The recirculated beam induces an HOM voltage which depends on the magnitude and direction of the beam displacement. Thus, the recirculated beam constitutes a feedback which can become unstable if the average beam current exceeds the threshold current.

For a two-pass accelerator with a single accelerating cavity containing a single dipole HOM with arbitrary polarization angle, $\alpha$, the transverse BBU threshold current is approximately given by the expression [4]

$$
\begin{aligned}
I_{\mathrm{th}} & =-\frac{2 c p_{b}}{e M^{*}(R / Q) Q_{L} k \sin \left(\omega T_{r}\right)} \\
M^{*} & \equiv M_{12} \cos ^{2} \alpha+\left(M_{14}+M_{32}\right) \sin \alpha \cos \alpha+M_{34} \sin ^{2} \alpha,
\end{aligned}
$$

where $p_{b}$ is the beam momentum at the HOM, $c$ is the speed of light, $e$ is the electron charge, $k$ is the wave number $(\omega / c)$ of the HOM, $(R / Q) Q_{L}$ is the shunt impedance of the HOM, $T_{r}$ is the recirculation time, and the $M_{i j}$ are the elements of the recirculation transport matrix from the cavity back to itself (which can describe coupled transverse motion). This equation is a good approximation only under the condition that $M^{*} \sin \left(\omega T_{r}\right)<0$ and $\omega T_{r} / 2 Q_{L} \ll 1$. If $M^{*} \sin \left(\omega T_{r}\right)$ is positive, Eq. (1) yields a negative threshold current which implies absolute beam stability. However, the beam can still go unstable at extremely high values of the beam current even if $M^{*} \sin \left(\omega T_{r}\right)>0$ [5]. This discrepancy is caused by the assumption that the voltage induced by the beam on the second pass is a small perturbation to the HOM voltage, which fails for beams with a large charge per bunch. The dependence of the threshold for positive values for $M^{*} \sin \left(\omega T_{r}\right)$ was predicted analytically and observed in simulations by others $[4,6,7]$.

Despite its simplicity, the single mode analytic model used in deriving Eq. (1) is applicable for the FEL. There are three primary reasons and they are based on rf measurements used to characterize HOMs. The first is that the bandwidths of the dangerous modes are (1-10) $\mathrm{kHz}$ whereas separation between polarizations is (0.51.0) MHz. Thus, the separation between polarizations is sufficiently large that they can be treated independently. Second, the loaded $Q$ s are an order of magnitude different between polarizations of a given dipole HOM. Consequently, only the dominant polarization needs to be considered. Third, from cavity to cavity modes are separated by $100 \mathrm{~s}$ of $\mathrm{kHz}$ up to several $\mathrm{MHz}$. Because of the frequency separation, modes do not destructively interfere and the threshold current is due to the worst individual HOM.

While preliminary measurements of BBU in the FEL Upgrade Driver were made in 2004, the work presented in this paper represents a more systematic approach to address many of the questions left unanswered by the previous work [2]. In particular, a better characterization of the instability itself was required to adequately benchmark existing BBU simulation codes. This required that the threshold current be accurately determined using several independent measurements and compared to simulation results. Additionally, the ability to successfully exercise a variety of BBU suppression techniques, by both appropriate modification of the beam optics and direct $Q$-damping methods, was of great importance.

\section{EXPERIMENTAL CHARACTERIZATION OF BBU}

The primary goal of these BBU studies was to characterize the instability to the extent that BBU simulation codes could be satisfactorily benchmarked. This required that the mode causing the instability be identified, the threshold current be accurately measured, and the optics of the FEL Driver be characterized.

\section{A. Mode identification}

The key element in the measurements used to characterize BBU was the fact that the zone 3 cryomodule, unlike previous CEBAF modules, uses DESY-like coaxial HOM couplers. Each cavity has two HOM couplers where cables connected to each port are loaded on $50 \Omega$ resistors. In order to monitor the HOM power, a small portion of the signal from each HOM port is directed to a Schottky diode by a $-20 \mathrm{~dB}$ directional coupler. The output of each diode is connected to a separate oscilloscope channel. This allows the HOM power to be individually monitored from each of the 8 cavities. Operating in continuous-wave (cw) mode, we slowly increased the average beam current until we observed exponential growth of the HOM power from cavity 7 which occurred simultaneously with a machine trip caused by excessive beam losses. This process of slowly ramping up the current was repeated several times to ensure that, indeed, the instability developed at the same current each time.

Upon identifying cavity 7 as containing the unstable mode, the next measurement was to identify the frequency of the mode. To do this, the signals from the HOM coupler are split further after the $-20 \mathrm{~dB}$ directional coupler, with one part connected to a Schottky diode to measure the power while the other part is sent directly to an oscilloscope to measure the voltage (a schematic of this setup is shown in Fig. 1). A screen shot of the oscilloscope showing the HOM power and voltage during BBU is given in Fig. 2. Taking the fast-Fourier transform (FFT) of the voltage 


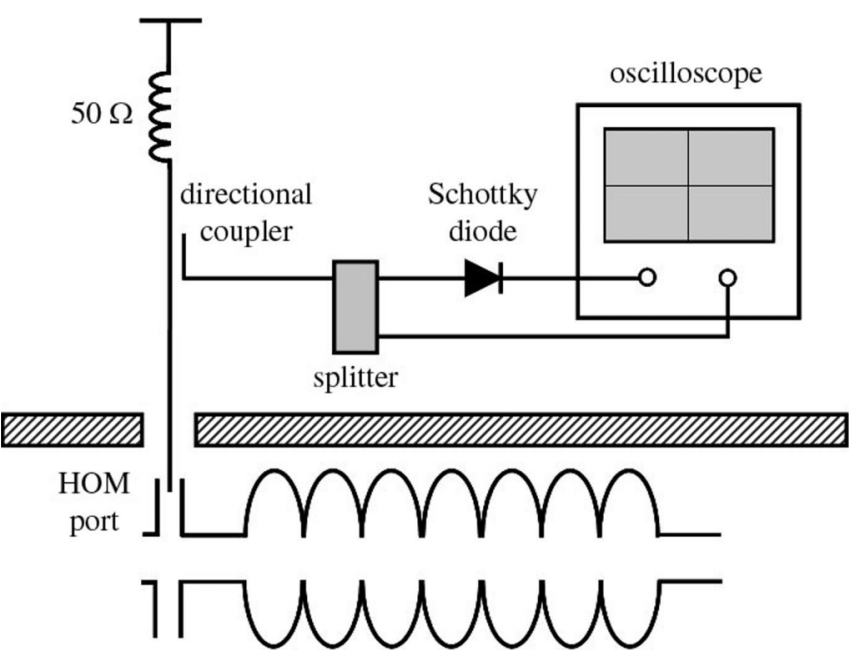

FIG. 1. Schematic of the experimental setup for simultaneously measuring the HOM power and voltage from a particular cavity.

signal reveals that the mode frequency is $2106.007 \mathrm{MHz}$ (see Fig. 3).

The results of our measurements show that with nominal, decoupled optics for an $88 \mathrm{MeV}$ machine configuration, the most dangerous mode is at a frequency of 2106.007 MHz and located in cavity 7. This is in agreement with simulation results which predict this mode is highly unstable [8]. Even before observing BBU directly, this mode was considered one of the prime candidates for causing BBU. Prior to these measurements, the first two dipole HOM passbands were measured for each of the 8 cavities in the zone 3 cryomodule and the $2106 \mathrm{MHz}$ mode has the second highest shunt impedance, $(R / Q) Q_{L}$, of the 224 modes measured. In fact, the highest impedance mode is also in cavity 7, at a frequency of $2116 \mathrm{MHz}$. However,

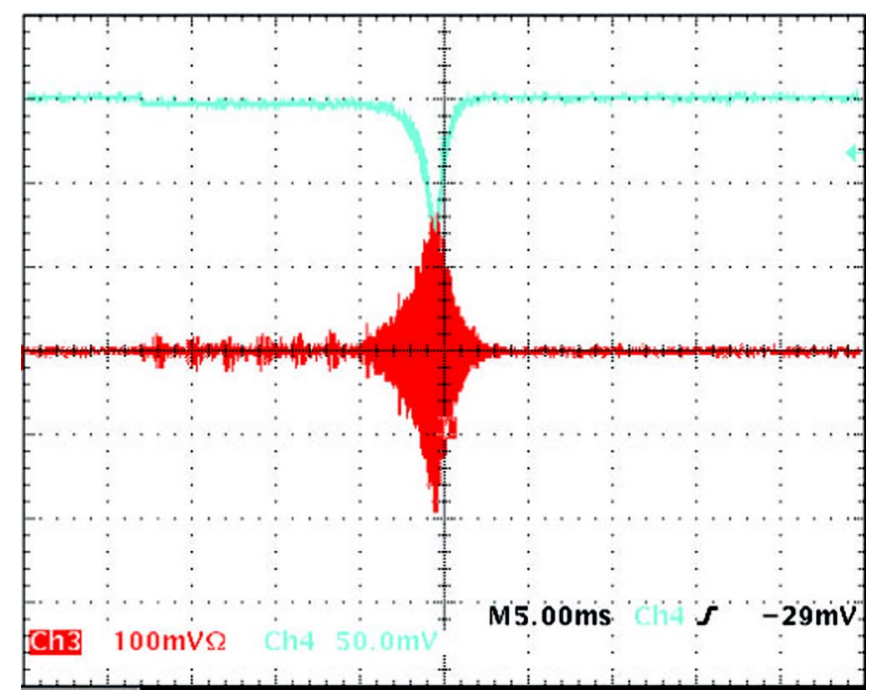

FIG. 2. (Color) A screen shot of an oscilloscope showing the HOM voltage (red) and power (blue) of the 2106.007 MHz HOM in cavity 7 of zone 3 during BBU.

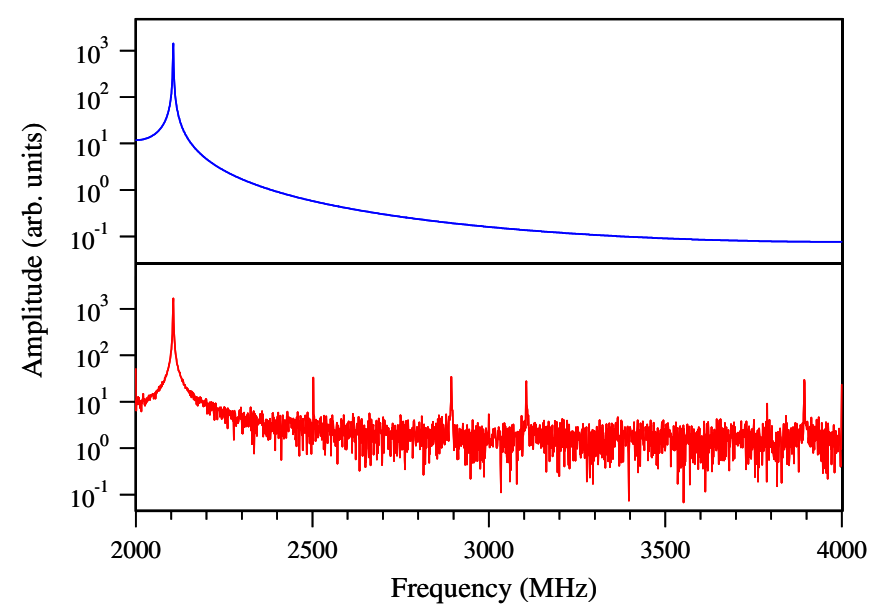

FIG. 3. (Color) FFT of a pure $2106.007 \mathrm{MHz}$ signal (top) and FFT of the HOM voltage from cavity 7 during BBU (bottom).

subsequent measurements confirmed that this HOM was not a threat for causing BBU because $M^{*} \sin \left(\omega T_{r}\right)>0$ for the mode.

During the onset of beam breakup due to $2106 \mathrm{MHz}$, the Schottky diodes connected to cavities 3 and 8 also detected a growth of the HOM power. Measuring the voltages from these cavities and taking the FFT of the signals yielded the frequencies 1786.206 and $1881.481 \mathrm{MHz}$ for cavities 3 and 8 , respectively. According to the results of earlier HOM measurements, these two modes have relatively low impedances and simulations predict that the threshold current due to these modes is at least an order of magnitude higher than that of the $2106 \mathrm{MHz}$ mode in cavity 7. It was discovered that the Schottky diode signals from cavities 3 and 8 had nearly the same growth rate as the signal from cavity 7 . This suggested that the other modes are being driven by the $2106 \mathrm{MHz}$ mode after it goes unstable. After the onset of BBU, the transverse beam displacement is deflected at the frequency of $2106.007 \mathrm{MHz}$. This frequency is aliased to sideband frequencies which, for a bunch frequency of $37.425 \mathrm{MHz}$, appear at $\pm 10.207 \mathrm{MHz}$ around the beam harmonics. As the instability grows the sidebands become sufficiently strong to the point that they are able to resonantly excite modes which lie at the sideband frequencies. To within a few tens of kilohertz (the error in our measurement of the frequencies), the $1786.206 \mathrm{MHz}$ mode corresponds to the lower sideband frequency of the 48th beam harmonic $(48 \times$ $37.425 \mathrm{MHz}-10.207 \mathrm{MHz}$ ) while the $1881.481 \mathrm{MHz}$ mode corresponds to the upper sideband frequency of the 50th beam harmonic $(50 \times 37.425 \mathrm{MH} z+10.207 \mathrm{MHz})$. This phenomenon of sidebands driving otherwise stable HOMs unstable was verified through simulations.

\section{B. Measuring the threshold current}

Given a description of the machine optics and HOM parameters (frequency, $Q_{L}, R / Q$, and polarization), $\mathrm{BBU}$ 
simulation codes calculate a threshold current for beam stability. Hence, the easiest way to benchmark the codes is to compare this threshold current with experimental measurements. Three different methods were used to measure the threshold current for a particular machine configuration.

The first, if the threshold current was sufficiently small, was by direct observation; that is, simply noting the average current from a beam current monitor at which a BBUinduced machine trip occurs. The remaining two methods make use of an important aspect of BBU, namely, how the HOM voltage behaves above and below the threshold current. The evolution of the voltage is described by the following equation [4]:

$$
V=V_{o} \exp \left(-\frac{\omega t}{2 Q_{L}} \frac{I_{\mathrm{th}}-I_{o}}{I_{\mathrm{th}}}\right) .
$$

From Eq. (2), one can extract a useful quantity which we define as the effective quality factor

$$
Q_{\mathrm{eff}}=\left(\frac{I_{\mathrm{th}}}{I_{\mathrm{th}}-I_{o}}\right) Q_{L} .
$$

This simple relation states that one needs only to measure the effective $Q$ as a function of the average beam current and, in principle, the threshold is easily extracted. Note that Eq. (3) is valid both above and below the threshold current.

The beam-transfer function (BTF) measurement is the second method used to measure the threshold current and amounts to using a network analyzer to make an $S_{21}$ measurement of a particular mode as a function of average beam current. By measuring the effective $Q$, that is, the quality factor of the combined HOM-beam system measured from the $-3 \mathrm{~dB}$ points of the frequency curve, as a function of current, Eq. (3) can be used to extract the threshold current.

The third and final measure of the threshold is achieved by measuring the growth rate of the HOM power while at currents above the threshold. With this method the growth rate is described by the time constant for the HOM-beam system

$$
\tau_{\mathrm{eff}}=\left(\frac{I_{\mathrm{th}}}{I_{\mathrm{th}}-I_{o}}\right) \tau_{o}
$$

where $\tau_{o}$ is the natural decay time of the HOM. Similar to the BTF measurement, Eq. (4) can be used to extract the threshold current after measuring the effective $\tau$ as a function of average beam current.

The method of measuring the HOM power and the BTF measurement combine to create a complementary set of measurements. Whereas measuring the power is a timedomain measurement made above the threshold current (and typically in pulsed-beam mode), the BTF measurement is inherently a frequency-domain measurement made below the threshold current (and typically with cw beam).

\section{Direct observation}

The most straightforward way to measure the threshold is to slowly increase the average beam current until the machine trips off due to excessive beam losses and the current goes to zero. The current just prior to the machine tripping represents the threshold current and was measured to be $2.3 \mathrm{~mA}$. The machine trip was simultaneously observed with an exponential growth in the HOM power to ensure that the instability, and not other beam loss mechanisms such as poor transmission, was the cause.

\section{Beam-transfer function measurement}

The BTF technique allows one to determine the BBU threshold for individual HOMs while doing the measurements below the threshold current. In earlier BBU experiments at the Jefferson Laboratory FEL Demo (the predecessor to the FEL Upgrade) described in [9], beam oscillations were excited at the injector using a stripline kicker [10]. The cavity response was measured at the frequency of the kicker signal through the cavity probe. The frequency of the signal was swept to measure the transfer function. Dangerous HOMs appeared as resonance peaks in the response signal during the frequency scan. The height of the resonance peaks of dangerous HOMs varied with the average beam current and a linear fit of the logarithm of the height of HOM resonance peaks plotted as a function of the logarithm of the beam current was used to determine the threshold. However, by measuring the $Q_{L}$ of the resonance curve and invoking Eq. (3), we can simplify the analysis a great deal because $1 / Q_{\text {eff }}$ is a linear function of the beam current.

Initial BTF measurements using the scheme described above kicked the beam at a frequency of $2106 \mathrm{MHz}$ with a stripline kicker and measured the $Q_{L}$ of the mode as a function of average beam current; plotting $1 / Q_{\text {eff }}$ versus the current results in the expected linear relationship. At the threshold current the quantity $1 / Q_{\text {eff }}$ becomes zero. Therefore, the point at which the linear fit intersects the current axis defines the threshold current. For the $2106 \mathrm{MHz}$ mode, this point occurs at $(2.3 \pm 0.1) \mathrm{mA}$ in agreement with the direct observation measurement.

Because of the accessibility to the HOM ports of the cavities in zone 3, we could simplify the BTF measurement substantially by exciting the beam directly through an HOM port of the cavity. The response signal was measured from the other HOM port of the same cavity. This technique had a significantly higher signal-to-noise ratio than the method used previously and eliminated the need for a kicker and a high-power amplifier. As mentioned, because the quality factor of the HOM resonance was measured as a function of the beam current instead of the height of resonance peaks, we significantly simplified the analysis of the data.

With the new experimental setup, the BTF of the $2106 \mathrm{MHz}$ mode was repeated. For this mode 


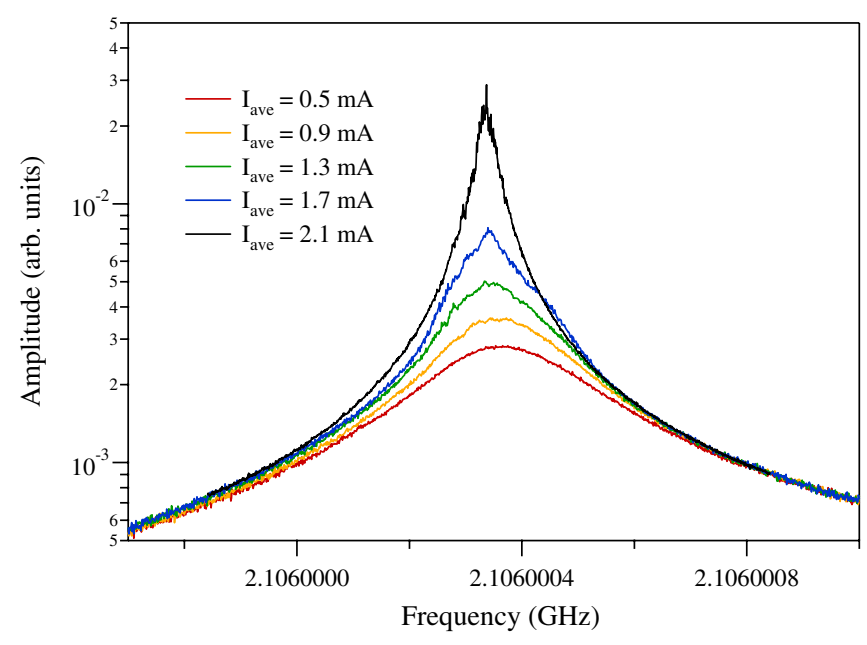

FIG. 4. (Color) The resonance curve for the $2106 \mathrm{MHz} \mathrm{HOM}$ as a function of average beam current with nominal, decoupled optics. Note that the effective $Q$ of the curve increases as the current increases. This indicates the system is unstable.

$M^{*} \sin \left(\omega T_{r}\right)<0$, and the height of the resonance peak grows and the quality factor increases with the beam current. The resonance curve data for different values of beam current are shown in Fig. 4. The $1 / Q_{\text {eff }}$ term is a linear function of the beam current and has a negative slope as displayed in Fig. 5. Analysis of the data yields a threshold current of $(2.4 \pm 0.1) \mathrm{mA}$ which is in excellent agreement with the results of the kicker-based BTF measurement.

The BTF technique is also useful in establishing whether a mode is stable or unstable by determining the sign of the term $M^{*} \sin \left(\omega T_{r}\right)$ which appears in the denominator of Eq. (1). Recall that Eq. (1) is applicable only if this term is less than zero. If it is greater than zero, the approxi-

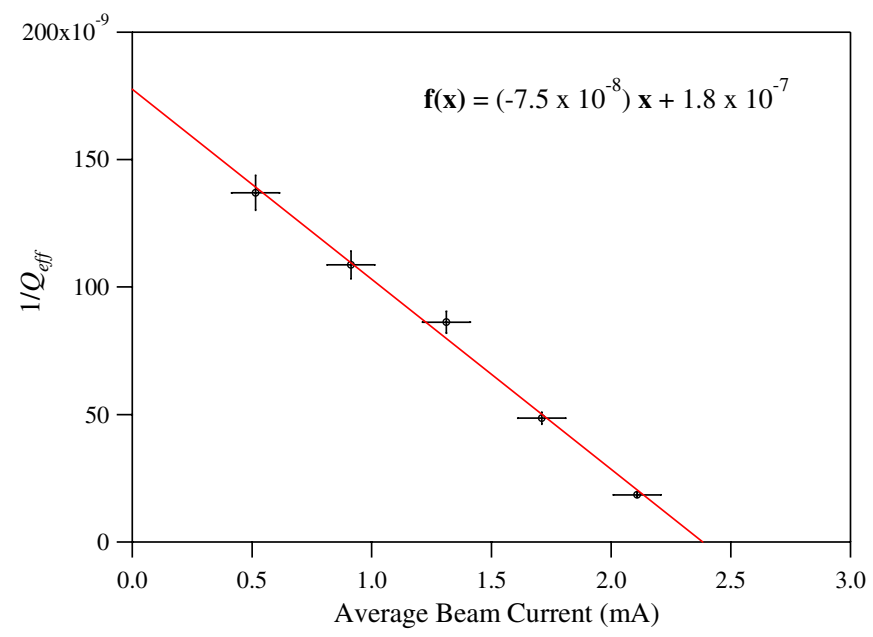

FIG. 5. (Color) A plot of $1 / Q_{\text {eff }}$ versus average beam current from the data in Fig. 4. The intersection of the least squares fit (functional form given on the plot) with the horizontal axis determines the threshold current to be $2.4 \mathrm{~mA}$.

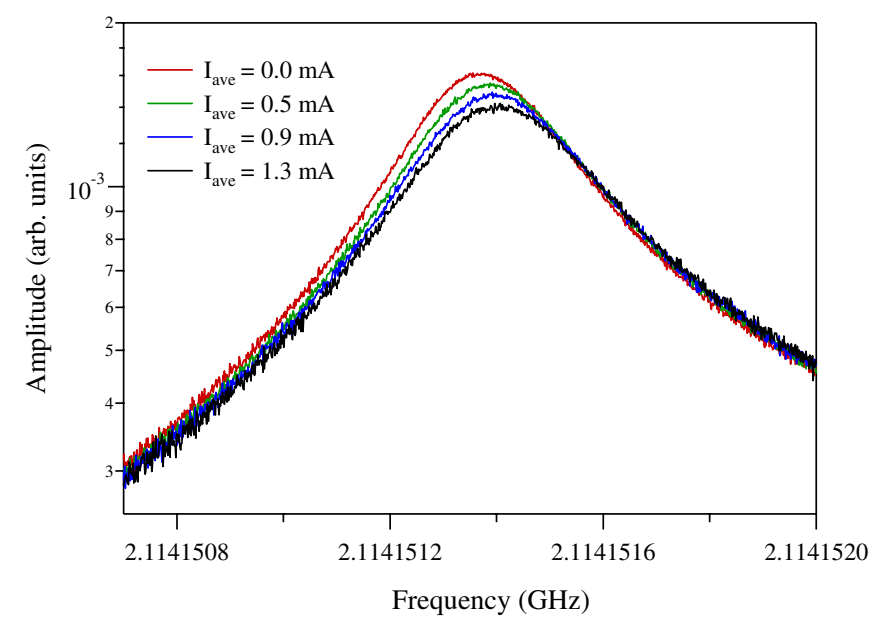

FIG. 6. (Color) The resonance curve for the $2114 \mathrm{MHz} \mathrm{HOM}$ as a function of average beam current with nominal, decoupled optics. Note that the effective $Q$ of the curve decreases as the current increases. This indicates the system is stable.

mations made in deriving Eq. (1) are no longer valid. Studies with BBU simulations show that, for cases where $M^{*} \sin \left(\omega T_{r}\right)>0$, the instability can still develop, but does so at currents of several Ampères [4]. (Note, the situation is different when $\omega T_{r} / 2 Q_{L}$ is no longer $\ll 1$, as is the case in some large-scale ERLs being planned [7].) This is referred to as the pseudostable regime, since for all practical purposes a threshold current on the order of an Ampère for the $10 \mathrm{~mA}$ FEL Upgrade means the system will be stable.

If $M^{*} \sin \left(\omega T_{r}\right)>0$, the slope of $1 / Q_{\text {eff }}$ changes its sign and becomes positive. In this case, the height of the resonance peak decreases and the quality factor becomes smaller as the beam current increases. This is illustrated in Fig. 6 which shows the results of the BTF measurement for the $2114 \mathrm{MHz}$ mode located in cavity 4 . The line $1 / Q_{\text {eff }}$ crosses the horizontal axis at a negative beam current.

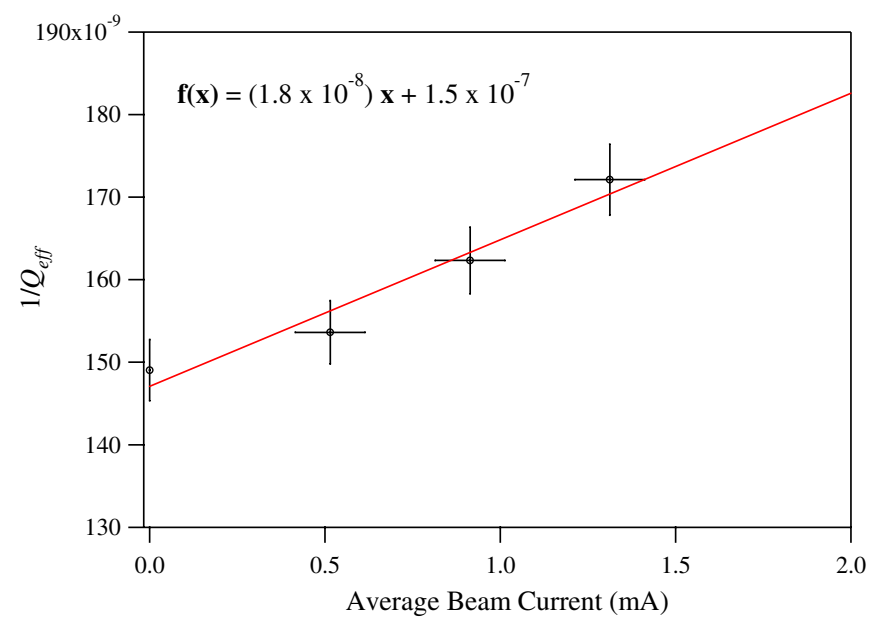

FIG. 7. (Color) A plot of $1 / Q_{\text {eff }}$ versus average beam current from the data in Fig. 6. The least squares fit is used to determine that the mode is stable. 
Figure 7 shows that the fit of the experimental data has a positive slope and crosses the horizontal axis at $-8.3 \mathrm{~mA}$, thus indicating the mode is stable with this particular optics configuration.

Because the $2116 \mathrm{MHz}$ mode in cavity 7 has the highest impedance of the measured HOMs in zone 3, a BTF measurement was performed to determine if the mode posed a threat for causing BBU. The results of the measurement show that $M^{*} \sin \left(\omega T_{r}\right)>0$ for the mode. The reason why $2106 \mathrm{MHz}$ is unstable and $2116 \mathrm{MHz}$ is stable can be readily explained from Eq. (1). Because the machine optics are decoupled, $M_{32}=M_{14}=0$, and both modes are vertically polarized, $\alpha=90^{\circ}$ (as determined by measurements discussed in a later section), $M^{*}$ reduces to $M_{34}$. From the model lattice the sign of $M_{34}$ is positive (for this discussion the magnitude is not important). It follows that, for the recirculation time of the FEL Driver, $433.199 \quad \mathrm{~ns}, \quad M_{34} \sin \left(\omega_{2106} T_{r}\right)<0 \quad$ and $2106.007 \mathrm{MHz}$ is unstable, whereas $M_{34} \sin \left(\omega_{2116} T_{r}\right)>0$ and $2116.585 \mathrm{MHz}$ is stable.

\section{Growth rate measurement}

Growth rate measurements were performed by measuring the HOM power from cavity 7 while operating the beam in pulsed mode. The macropulse length was chosen to be sufficiently long and the current to be sufficiently large such that BBU would develop within the macropulse. By fitting the rise $\left(\tau_{\text {eff }}\right)$ and decay $\left(\tau_{o}\right)$ time of the instability, Eq. (4) can be rearranged and used to solve for the threshold current explicitly

$$
I_{\mathrm{th}}=\left(\frac{\tau_{\mathrm{eff}}}{\tau_{\mathrm{eff}}-\tau_{o}}\right) I_{o}
$$

These measurements were performed with macropulse currents of 3.0, 3.5, and $4.1 \mathrm{~mA}$ and yielded threshold currents of $(2.3 \pm 0.2),(2.3 \pm 0.1)$, and $(2.3 \pm 0.1) \mathrm{mA}$, respectively. Additionally, the $Q_{L}$ of the $2106 \mathrm{MHz}$ mode, extracted from the fit of the decay time, is within $7 \%$ agreement of previous rf measurements. Figure 8 shows the rise and decay times of the instability for all three macropulse currents plotted on the same graph (note the vertical logarithmic scale). This nicely illustrates both the exponential growth of HOM power during BBU and also how the instability growth time depends on current. Note that the decay times are identical, as they should be, as this represents the natural decay time of the $2106 \mathrm{MHz}$ mode that caused the instability.

An alternate way of extracting the threshold current is to plot the three values of $1 / \tau_{\text {eff }}$ against the macropulse current and fit the data with a line in the same way as the BTF measurements. Finding the intersection of the extrapolated linear fit and the current axis indicates that the threshold current is $(2.2 \pm 0.2) \mathrm{mA}$ as shown in Fig. 9.

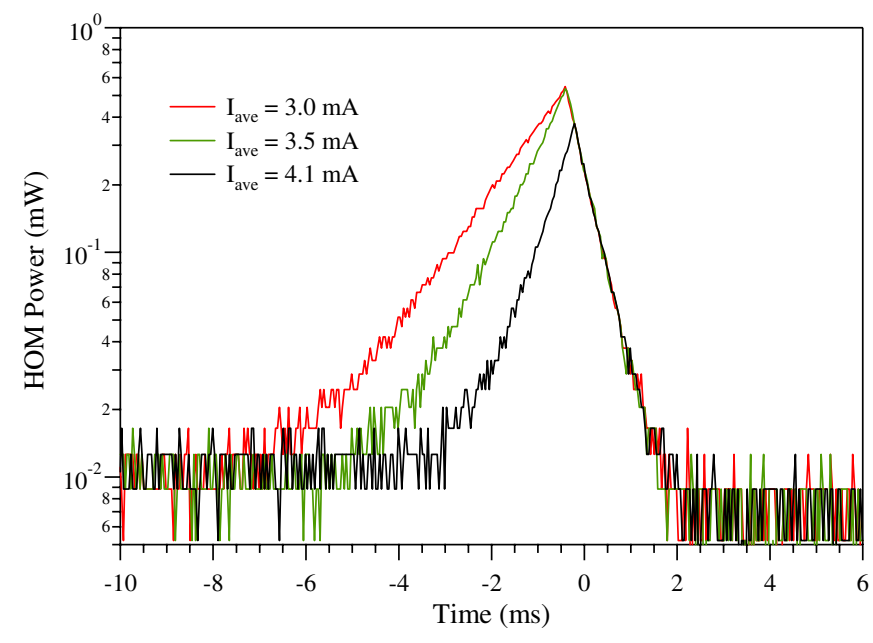

FIG. 8. (Color) A plot of the HOM power of the $2106 \mathrm{MHz}$ mode as a function of time for three different values of macropulse current (note the logarithmic scale of the vertical axis).

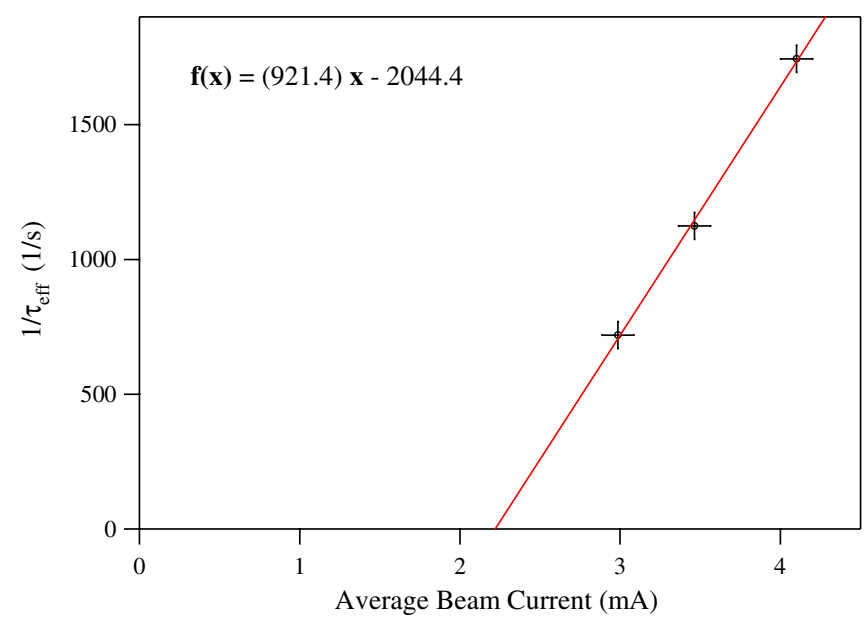

FIG. 9. (Color) A plot of the three values of $1 / \tau_{\text {eff }}$ corresponding to each macropulse current from Fig. 8 versus the macropulse current. The threshold current is $2.2 \mathrm{~mA}$ and is extracted in the same manner as the BTF measurements.

\section{Measuring HOM polarization}

In light of Eq. (1), an important parameter in characterizing HOMs is the polarization of the modes. Before these values were known, BBU simulations were performed with dipole $\mathrm{HOM}$ pairs assigned orientations of $0^{\circ}$ and $90^{\circ}$ and then repeated with orientations of $90^{\circ}$ and $0^{\circ}$, with the threshold taken as the lowest of the two cases. Essentially only worst case scenarios were simulated. In principle, bead pull measurements can be used to extract mode polarizations. However, the small geometric perturbations from cavity to cavity, introduced during the fabrication process, lead to a unique HOM spectrum for each cavity. Consequently, depending on the extent of the perturbations, the same mode can be oriented differently from one cavity 
to the next. Therefore, it becomes necessary to use beambased methods to accurately assess HOM polarizations.

The measurement required that only the first pass beam be transported through the linac. To prevent the second pass (energy recovered) beam from propagating through the linac, the beam was directed to an insertable dump in the recirculator. Because it is a low power dump it could only tolerate tune-up beam ( $250 \mu$ s long macropulses with a $4.678 \mathrm{MHz}$ bunch repetition rate every $2 \mathrm{~Hz}$ with a charge per bunch of $135 \mathrm{pC}$ ). When the beam passed through a cavity, it excited cavity HOMs. The voltage of dipole HOMs induced by a beam pulse depended on a number of beam and HOM parameters such as the bunch repetition rate, pulse length, and the HOM frequency. Most importantly for our measurements was that the voltage of dipole HOMs depends linearly on the beam displacement in the cavity.

The beam was displaced in each plane independently using either an upstream vertical corrector or a horizontal corrector. The corrector was changed by $\pm 150 \mathrm{G}-\mathrm{cm}$, in increments of $50 \mathrm{G}-\mathrm{cm}$, from its nominal setpoint while the response of the HOM of interest was measured by a network analyzer (NWA), zero spanned at the frequency of a chosen HOM. To ensure that only the voltage of the chosen HOM was measured the intermediate frequency (IF) bandwidth of the NWA was limited to $30 \mathrm{kHz}$. Note that only the input of the NWA was used to measure the signal while the output was terminated. Therefore, a spectrum analyzer could be used instead of the NWA. This process was repeated for 8 of the most dangerous HOMs in zone 3, taking care to measure each pair of the dipole modes.

The beam induced voltage for a dipole HOM goes linearly with the off-axis displacement of the beam. By exciting the mode with the beam displaced either horizontally or vertically, the HOM polarization can be found according to

$$
\alpha=\tan ^{-1}\left(\frac{V_{y}}{V_{x}}\right)
$$

where $V_{y}$ and $V_{x}$ are the voltage responses of the HOM due to a vertical and horizontal displacement, respectively, and are extracted from fits of the measured data. The measured data for the $2106 \mathrm{MHz}$ mode is displayed in Fig. 10 and a summary of the measurements for all the HOMs are given in Table I. Within each dipole HOM, the two polarizations are separated in frequency by a few hundred $\mathrm{kHz}$, making it possible to excite each independently. In addition, one polarization typically has a loaded $Q$ an order of magnitude larger than its partner. It was found that these high $Q$ modes are oriented approximately vertically, thereby making BBU less of a threat in the horizontal plane (see Table I).

We point out that two modes exist which do not appear to be orthogonal, as expected. They are the $4 \pi / 7$ mode in cavity 4 and the $3 \pi / 7$ mode in cavity 3 . The reason for this is unclear and they are still under investigation.

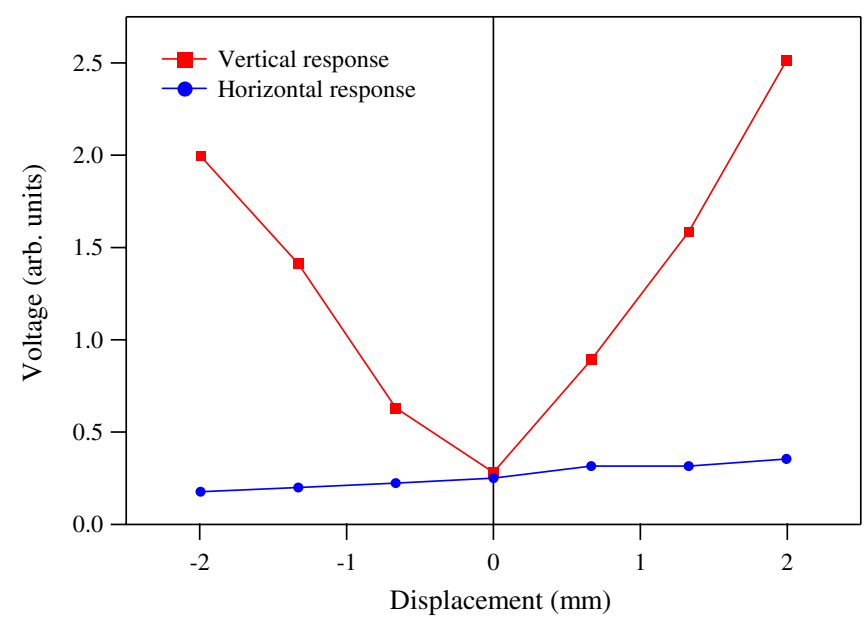

FIG. 10. (Color) Measured response of the $2106 \mathrm{MHz} \mathrm{HOM}$ due to vertical (red) and horizontal (blue) displacements through cavity 7 .

TABLE I. Results of beam-based measurements to determine HOM polarizations of several of the most dangerous modes. For each dipole HOM pair, the higher $Q_{L}$ mode was oriented approximately vertically.

\begin{tabular}{l|lccc}
\hline \hline \multirow{3}{*}{ Cavity } & Mode & $\begin{array}{c}\text { Frequency } \\
(\mathrm{MHz})\end{array}$ & $\begin{array}{c}Q_{L} \\
\left(10^{6}\right)\end{array}$ & $\begin{array}{c}\text { Orientation } \\
\text { (degrees) }\end{array}$ \\
\hline 8 & $\mathrm{TM}_{110}$ & 2102.591 & 2.6 & $84 \pm 11$ \\
& $3 \pi / 7$ & 2103.009 & 0.2 & $8 \pm 13$ \\
\cline { 2 - 5 } & $\mathrm{TM}_{110}$ & 2113.346 & 3.1 & $80 \pm 2$ \\
& $4 \pi / 7$ & 2114.154 & 0.4 & $11 \pm 2$ \\
\hline 7 & $\mathrm{TM}_{110}$ & 2105.999 & 6.1 & $88 \pm 2$ \\
& $3 \pi / 7$ & 2106.697 & 0.3 & $4 \pm 1$ \\
\cline { 2 - 5 } & $\mathrm{TM}_{110}$ & 2116.583 & 6.7 & $86 \pm 10$ \\
& $4 \pi / 7$ & 2117.225 & 0.5 & $4 \pm 1$ \\
\hline \multirow{4}{*}{3} & $\mathrm{TM}_{110}$ & 2102.537 & 0.3 & $25 \pm 1$ \\
& $3 \pi / 7$ & 2102.642 & 0.6 & $63 \pm 1$ \\
\cline { 2 - 5 } & $\mathrm{TM}_{110}$ & 2113.991 & 0.4 & $33 \pm 1$ \\
& $4 \pi / 7$ & 2114.151 & 5.2 & $87 \pm 6$ \\
\hline & $\mathrm{TM}_{110}$ & 2104.201 & 2.5 & $38 \pm 1$ \\
& $3 \pi / 7$ & 2104.408 & 0.3 & $14 \pm 1$ \\
\cline { 2 - 5 } & $\mathrm{TM}_{110}$ & 2115.386 & 2.9 & $75 \pm 1$ \\
& $4 \pi / 7$ & 2115.683 & 0.4 & $18 \pm 2$ \\
\hline \hline
\end{tabular}

\section{Beam optics}

To benchmark the BBU codes, it is important that the beam optics used in the simulations accurately describes the optics of the machine that the measurements were performed on. For each machine configuration we have a record, or "all-save," of the important machine settings. That is to say, we know the quadrupole and dipole strengths, the accelerating gradient for each cavity, the 
TABLE II. Comparison of experimental measurements, simulation results, and the analytic formula for determining the threshold current.

\begin{tabular}{llc}
\hline \hline & Method & Threshold current (mA) \\
\hline Simulation & MATBBU & 2.1 \\
& TDBBU & 2.1 \\
& New code & 2.1 \\
& BI & 2.1 \\
\hline Experimental & Direct observation & $2.3 \pm 0.2$ \\
& Kicker-based BTF & $2.3 \pm 0.1$ \\
& Cavity-based BTF & $2.4 \pm 0.1$ \\
& Growth rates & $2.3 \pm 0.2$ \\
& (method 1) & $2.3 \pm 0.1$ \\
& & $2.3 \pm 0.1$ \\
& (method 2) & $2.2 \pm 0.2$ \\
\hline Analytic & Analytic formula & 2.0 \\
\hline \hline
\end{tabular}

linac phasing, and the injection energy. This represents all the information required to reconstruct the optics in the BBU simulations. While not determined experimentally, this represents a good starting point. The results of simulations based on the all-save data to describe the beam optics are displayed in Table II and will be discussed in the following section.

\section{COMPARISON OF EXPERIMENTAL DATA WITH SIMULATION RESULTS}

A comparison of the experimental measurements to determine the threshold current and predictions from simulations are displayed in Table II.

The simulations were performed with the three BBU codes developed at Jefferson Laboratory; TDBBU [11,12], MATBBU [6,13], and a more recently developed code [4] as well as a code developed at Cornell University called BI [14]. For simplicity all the codes were run with the HOM kicks placed before each accelerating cavity. As expected, the predictions from all four codes agree.

We utilized a variety of experimental techniques to measure the threshold current and they all show excellent agreement amongst themselves. The BTF measurement used a $\mathrm{cw}$ beam operating at currents below the threshold current, while the growth rate measurements employed pulsed beam operating at currents above the threshold. Thus, under a variety of beam conditions (cw and pulsed) and operating in different current regimes (above and below the threshold), there is complete agreement in determining the threshold current.

To emphasize the value of Eq. (1), Table II includes the threshold current as determined by plugging values for the $2106 \mathrm{MHz}$ mode in Eq. (1). The analytic formula and the simulations show excellent agreement.

\section{BBU SUPPRESSION: BEAM OPTICAL CONTROL}

With a comprehensive characterization of BBU complete, our attention is now turned towards exercising suppression techniques. These techniques can be divided into two categories. The first involves manipulating the transverse beam optics in such a way as to reduce the coupling between the recirculated beam and cavity HOMs. The second is to apply feedback to the cavity, and will be discussed in the following section.

Methods to manipulate the transverse beam optics in order to suppress BBU were first discussed by Rand and Smith in 1980 [15]. Following the suggestions presented in that work, we demonstrated the ability to raise the threshold current by use of point-to-point focusing, a local reflector, and a rotation. For a more complete introduction to these beam optical techniques, the reader is referred to Refs. [2,15-18].

\section{A. Point-to-point focusing}

With a change in the betatron phase advance, one can achieve point-to-point focusing $\left(M_{12}\right.$ or $\left.M_{34}=0\right)$ at the location of the cavity containing an unstable mode so that an HOM-induced kick on the first pass results in a zero displacement on the second pass. From measuring HOM polarizations, we know that the dangerous $2106 \mathrm{MHz}$ HOM is vertically polarized. To vary the vertical phase advance, the strengths of four vertically focusing quadrupoles in the recirculator were changed from their nominal setpoint in steps of $100 \mathrm{G}$ from $-200 \mathrm{G}$ to $+300 \mathrm{G}$. For a periodic FODO channel with $90^{\circ}$ phase advance per cell, if the focusing perturbations are uniformly applied over an integral number of betatron wavelengths, the betatron phases can be varied while minimally affecting the beam envelopes [19]. Changing the strengths of vertically focusing quadrupoles produces a significant shift in vertical phase advance, but only a modest shift in the horizontal (the reverse is true if changes are applied to the horizontally focusing quadrupoles).

For each change in the quadrupole strengths, the threshold current was measured either by direct observation (if the threshold current was sufficiently small) or by the BTF measurement. The effect of changing the phase advance is illustrated in Fig. 11 where the threshold current is plotted against the change in quadrupole strength. The results are quite dramatic; the threshold went from being less than $1 \mathrm{~mA}(-200 \mathrm{G})$ to the mode being stabilized $(+300 \mathrm{G})$. In fact, with the mode stabilized, we attempted a high average current run. We were eventually limited to $6 \mathrm{~mA}$ (due to dangerously high pressure spikes in the injector) with no indications of BBU.

The threshold current in Fig. 11 exhibits a dependence which is explained by the fact that the $M_{34}$ element of the recirculation matrix is proportional to $\sin \psi$ where $\psi$ is the betatron phase advance for a single recirculation from the 


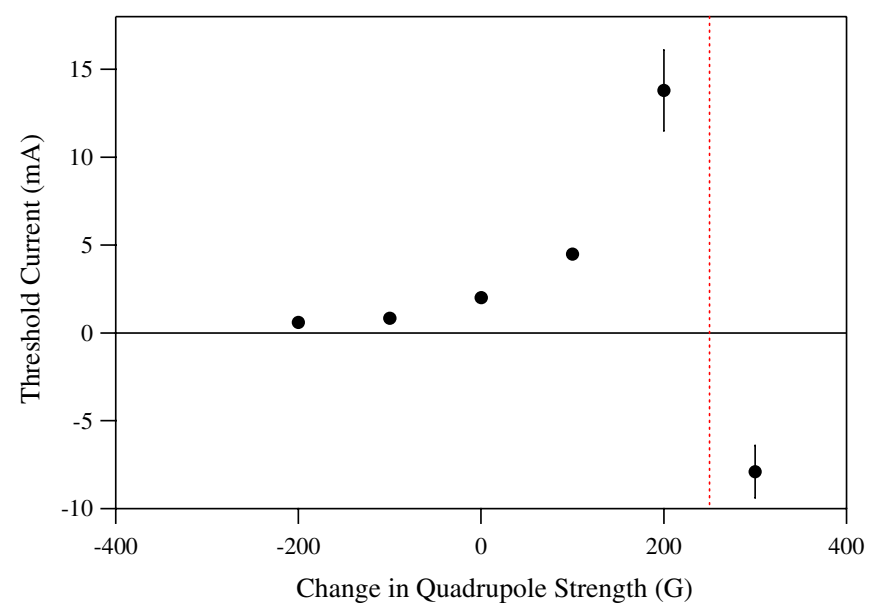

FIG. 11. (Color) A plot of the threshold current versus the change in quadrupole strength showing the effect of point-topoint focusing. At approximately $250 \mathrm{G}$ the change in phase advance makes the $M_{34}$ element of the recirculation matrix from the cavity back to itself equal to zero before changing sign and hence the threshold current to be negative.

cavity back to itself. For a quadrupole strength of approximately $250 \mathrm{G}$, the phase advance from cavity 7 back to itself is equal to $n \pi$, where $n$ is an integer number. Therefore $M_{34}$ can be expressed as

$$
M_{34} \propto \sin \psi=\sin (n \pi+\delta)=(-1)^{n} \sin \delta,
$$

where $\delta$ is the variation of the phase advance from $n \pi$. It follows that for small variations, $\delta$ is proportional to the change of the quadrupole strength. For the quadrupole strength +300 , the BTF measurements yielded a negative threshold. This indicates that $M_{34}$ changed its sign and the product $M^{*} \sin \left(\omega T_{r}\right)$ is positive.

It should be noted that using point-to-point focusing to suppress BBU proved to be particularly effective because the dangerous HOMs in the FEL Upgrade are localized in a single cryomodule. For an extended linac containing many dangerous modes, it may not be advantageous to modify the phase advance. While one mode may be made stable, in all likelihood the resulting change in phase advance will have harmful effects on other modes which were previously stable. Care should be taken when applying this method to large-scale ERLs with extended linacs.

\section{B. Local reflection}

The idea behind implementing a local reflector is to map a BBU-induced vertical kick into the horizontal plane, and likewise to map a BBU-induced horizontal kick to the vertical plane. A practical implementation of a local reflector using skew quadrupoles has been noninvasively embedded in the recirculator of the FEL Upgrade Driver. Operationally, normal quadrupoles upstream and downstream of the module are used as betatron matching telescopes. These allow transverse matching of the phase spaces across the reflector so that the module remains transparent to the rest of the machine [20]. To experimentally measure the effect of the reflector, we first established that the threshold current was $1.8 \mathrm{~mA}$ and caused by the $2106 \mathrm{MHz}$ mode without the reflector activated. The reflector was then activated and operation with $5.0 \mathrm{~mA}$ of average beam current was achieved with no indications of BBU. Because direct observation was not possible, a BTF measurement of the $2106 \mathrm{MHz}$ mode was performed. The results of the measurement yielded a new threshold current of $(9.2 \pm 0.4) \mathrm{mA}$; an increase by a factor of 5.1 . Additional BTF measurements were performed for the $2116 \mathrm{MHz}$ mode (also located in cavity 7) and the $2114 \mathrm{MHz}$ mode (cavity 4). The concern was that, by producing a reflection in the betatron planes, these modes which were stable for decoupled optics may become unstable. However, the results of the measurements show that the modes were stable and do not pose a threat for causing the instability.

\section{Rotation}

While the local reflector proved to be effective in increasing the threshold current, even stronger suppression can be achieved by generating a $90^{\circ}$ rotation of the betatron planes from cavity 7 back to itself. The $4 \times 4$ recirculation transfer matrix is then completely coupled and the off-diagonal $2 \times 2$ matrices are of opposite sign. The existing local reflector embedded in the FEL Driver recirculator can, in principle, be made to produce such a transfer matrix by changing the vertical betatron phase advance [21]. Upon changing the phase advance and generating a recirculation matrix that describes a rotation, we followed the usual procedure of comparing the extrapolated threshold current from the BTF measurement for the coupled and nominal beam optics. The result of the measurement for the $2106 \mathrm{MHz}$ mode shows that the mode has been stabilized. The slope of the line that fits the inverse of the effective $Q$ versus beam current data is positive. Extrapolating the line until it crosses the current axis yields a threshold current is $-17.0 \mathrm{~mA}$. This once again demonstrates the attractiveness of the BTF measurement which allows us to determine the threshold current while making measurements at currents where the beam is stable.

\section{BBU SUPPRESSION: FEEDBACK MECHANISMS}

Damping the HOM quality factors is achieved primarily by HOM couplers. However, because the damping in the zone 3 cavities is insufficient to prevent BBU, two methods to provide further damping were developed and successfully exercised in the FEL Upgrade Driver.

\section{A. Mode-by-mode, narrowband, cavity-based feedback}

The idea of the damping circuit is as follows; couple voltage from one of the HOM ports and using a narrow- 


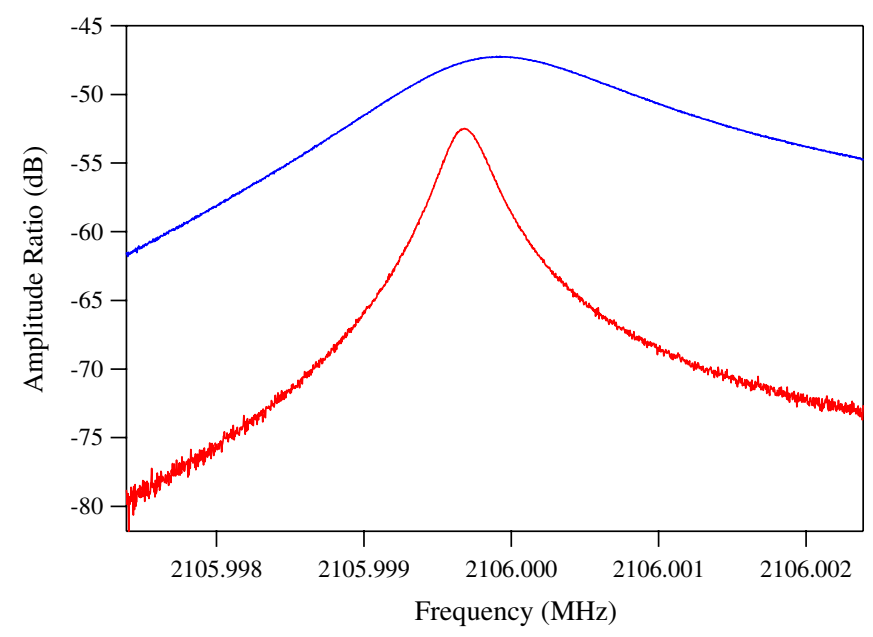

FIG. 12. (Color) The effect on the $Q$ of the $2106 \mathrm{MHz}$ mode with the cavity-based, narrowband feedback off (red curve represents $Q=6.2 \times 10^{6}$ ) and on (blue curve represents $Q=$ $\left.1.3 \times 10^{6}\right)$.

band filter select the HOM frequency of interest, shift the signal by $180^{\circ}$ in phase, amplify the signal, and then return it to the cavity through the same HOM port. By adjusting the gain and phase shift, we were able to reduce the HOM quality factor by a factor of 20 . However, if the suppression exceeded a factor 5 , the feedback became sensitive to disturbances such as the motion of cables and the amplifier warming up, making operation difficult.

With the damping circuit off, the threshold current was determined to be $2.3 \mathrm{~mA}$ by direct observation. After the feedback circuit was set to reduce the $Q_{L}$ of the $2106 \mathrm{MHz}$ mode by a factor of 4.8 (Fig. 12), the beam was turned on and the BBU threshold as determined by the BTF method was $(7.6 \pm 0.2) \mathrm{mA}$.

\section{B. 3-Stub tuners}

The 3-stub tuner acts as an impedance transformer. A stub tuner was attached to each cable connected to the two HOM ports of cavity 7 . The idea is to manipulate the stubs in such a fashion that the incident HOM voltage is reflected with a $180^{\circ}$ phase shift. Because the stub tuner is a passive device, the attenuation in the cables from the HOM ports became a factor and prevented optimal $Q$-damping. Nevertheless, a modest decrease (a factor of 2) in the $Q_{L}$ of the $2106 \mathrm{MHz}$ mode and the corresponding increase in the threshold current were observed.

\section{DISCUSSION OF SUPPRESSION TECHNIQUES}

Several methods for suppressing beam breakup have been successfully demonstrated in the FEL Upgrade Driver. A summary of the techniques and their effect on the $2106 \mathrm{MHz}$ mode is given in Table III.

While it is clear that direct $Q$-damping can increase the threshold by factors of a few, it could not completely
TABLE III. Summary of the BBU suppression techniques applied and their effect on the threshold current produced by the $2106 \mathrm{MHz}$ mode.

\begin{tabular}{lc}
\hline \hline Suppression technique & Effect on $2106 \mathrm{MHz}$ mode \\
\hline Damping circuit & $3.3 \times I_{\mathrm{th}}$ \\
3-stub tuner & $1.6 \times I_{\mathrm{th}}$ \\
Point-to-point focusing & Stabilized \\
Local reflector & $5.1 \times I_{\mathrm{th}}$ \\
Rotation & Stabilized \\
\hline \hline
\end{tabular}

stabilize the mode. Nonetheless, one of the attractive features of this technique is that it does not interfere with the beam optics.

The beam optical suppression techniques proved to be very effective at increasing the threshold current. In fact, we were able to stabilize the $2106 \mathrm{MHz}$ mode using pointto-point focusing and a rotation. However, as was discussed previously, point-to-point focusing cannot be arranged for each individual cavity in an accelerator with an extended linac. Therefore the usefulness of this method may be restricted to smaller machines or machines where dangerous HOMs are well localized.

The local reflector is routinely used in beam operations at the Jefferson Laboratory FEL to achieve high average currents. In July 2004, the reflector was activated when the FEL achieved $10 \mathrm{~kW}$ of laser power at 5.7 microns by running 1-second macropulses at a repetition rate of $0.25 \mathrm{~Hz}$ [3]. Despite the success of the local reflector, caution should be exercised when intentionally introducing strong betatron coupling. While it is possible to manipulate the transfer matrix to create reflections and rotations, optical mismatch must be corrected as it can generate beam loss that limits machine performance just as readily as BBU.

\section{CONCLUSION}

The Jefferson Laboratory FEL Upgrade Driver has proved to be a valuable test bed in investigating the multipass beam breakup instability and methods of suppression. The primary goal of these studies was to characterize BBU to the extent that BBU simulation codes could be benchmarked. The excellent agreement between the results of the four different simulation codes with a variety of experimental techniques leaves no doubt simulations can be used with confidence for predicting the threshold current of a particular machine with an accuracy of better than $10 \%$. This work represents the first time the BBU codes have been experimentally benchmarked. Equally important, the experimental results have confirmed the validity of the single mode analytic model of beam breakup.

Techniques to accurately measure the threshold current were demonstrated. The BTF measurement proved to be particularly useful as it provides a straightforward method for extracting the threshold current for a given machine 
configuration while working at currents where the beam is stable.

Additionally, two classes of suppression techniques were explored; beam optical schemes and direct $Q$-damping. It was demonstrated experimentally that, to varying degrees, all the suppression techniques were successful in increasing the threshold current for instability. The beam optical suppression techniques proved to be so effective, in fact, that invoking the local reflector has become part of the normal operation of the FEL Upgrade Driver.

\section{ACKNOWLEDGMENTS}

The authors gratefully acknowledge the FEL team for their support for the duration of the beam studies time. Additionally, we wish to thank Curt Hovater, Tom Powers, Chris Slominski, and Byung Yunn for their help and advice, and to Geoffrey Krafft for his careful reading of the manuscript. This work supported by The Office of Naval Research, the Joint Technology Office, NAVSEA PMS405, the Air Force Research Laboratory, U.S. Army Night Vision Lab, the Commonwealth of Virginia, NSF Cooperative Agreement No. PHY-9809799, and by DOE Contract No. DE-AC05-84ER40150.

[1] L. Merminga, D. R. Douglas, and G. A. Krafft, Annu. Rev. Nucl. Part. Sci. 53, 387 (2003).

[2] C. D. Tennant, K. B. Beard, D. R. Douglas, K. C. Jordan, L. Merminga, E. G. Pozdeyev, and T. I. Smith, Phys. Rev. ST Accel. Beams 8, 074403 (2005).

[3] S. V. Benson, D. R. Douglas, M. Shinn, K. B. Beard, C. Behre, G. Biallas, J. Boyce, H.F. Dylla, R. Evans, A. Grippo, J. Gubeli, D. Hardy, C. Hernandez-Garcia, K. C. Jordan, L. Merminga, G. R. Neil, J. Preble, T. Siggins, R. Walker, G. P. Williams, B. Yunn, S. Zhang, and $\mathrm{H}$. Toyokawa, in Proceedings of the 26th International
Free Electron Laser Conference, Trieste, Italy (Comitato Conferenze Elettra, 2004), pp. 229-232.

[4] E. G. Pozdeyev, Phys. Rev. ST Accel. Beams 8, 054401 (2005).

[5] G. A. Krafft, J. J. Bisognano, and S. Laubach (unpublished).

[6] B.C. Yunn, in Proceedings of the 1991 Particle Accelerator Conference, San Francisco, CA (IEEE, Piscataway, NJ, 1991), pp. 1785-1787.

[7] G. H. Hoffstaetter and I. V. Bazarov, Phys. Rev. ST Accel. Beams 7, 054401 (2004).

[8] C. D. Tennant, E. G. Pozdeyev, S. Simrock, A. Sun, and H. Wang, JLAB Technical Report No. TN-04-008, 2004.

[9] L. Merminga, I. Campisi, D. R. Douglas, G. A. Krafft, J. Preble, and B.C. Yunn, in Proceedings of the 2001 Particle Accelerator Conference, Chicago, IL (IEEE, Piscataway, NJ, 2001), pp. 173-175.

[10] N.S. R. Sereno, Ph.D. thesis, University of Illinois at Urbana-Champaign, 1994.

[11] G. A. Krafft and J. J. Bisognano, in Proceedings of the 1987 Particle Accelerator Conference, Washington, DC (IEEE Catalog No. 87CH2387-9), pp. 1356-1358.

[12] K. B. Beard, JLAB Technical Report No. TN-02-045, 2002.

[13] K.B. Beard, JLAB Technical Report No. TN-02-044, 2002.

[14] I. V. Bazarov and G. H. Hoffstaetter, in Proceedings of the 2004 European Particle Accelerator Conference, Lucerne, Switzerland, 2004, pp. 2194-2196.

[15] R. E. Rand and T. I. Smith, Part. Accel. 11, 1 (1980).

[16] E. G. Pozdeyev, J. J. Bisognano, R. Hajima, M. Sawamura, T. I. Smith, and C. D. Tennant, Nucl. Instrum. Methods Phys. Res., Sect. A 557, 176 (2006).

[17] B. C. Yunn, Phys. Rev. ST Accel. Beams 8, 104401 (2005).

[18] G. H. Hoffstaetter, I. V. Bazarov, and C. Song, Phys. Rev. ST Accel. Beams (to be published).

[19] D. R. Douglas, JLAB Technical Report 04-017, 2004.

[20] D. R. Douglas, JLAB Technical Report 04-016, 2004.

[21] D. R. Douglas, JLAB Technical Report 04-025, 2004. 